Solid State Ionics 20 (1986) 31-44

North-Holland, Amsterdam

\title{
A NONLINEAR LEAST SQUARES FIT PROCEDURE FOR ANALYSIS OF IMMITTANCE DATA OF ELECTROCHEMICAL SYSTEMS
}

\author{
Bernard A. BOUKAMP \\ Department of Chemical Technology, Laboratory of Inorganic Chemistry and Materials Science, \\ Twente University of Technology, P.O. Box 217, 7500 AE Enschede, The Netherlands
}

Received 15 July 1985

\begin{abstract}
A Nonlinear Least Squares Fit (NLLSF) program is described, with which frequency dispersion data of electrochemical systems can be analyzed in terms of an equivalent circuit. The NLLSF procedure uses a combination of an analytical and gradient search according to the Marquardt algorithm. Through the use of an unique Circuit Description Code (CDC) different equivalent circuits may be used with the program. The use of an analytical derivatives routine enhances the execution speed. The power of such a fit procedure is demonstrated in multi parameter fits, on synthetic and real data, performed by the program "EQIVCT".
\end{abstract}

\section{Introduction}

Frequency dispersion measurements (or impedance spectroscopy) have become a common technique for the study of mass and charge transport in electrochemical systems. With the availability of automated high quality frequency response analysis systems immittance (i.e. impedance or admittance) measurements can be obtained more easily then before, using hand-balanced bridges. Especially in the field of solid electrolyte research these measurements have been used widely in the study of ionic conduction, grain boundary impedance, and electrolyte/electrode interface characteristics [1-6]. Recently this measurement technique has been used successfully in diffusion measurements on mixed conducting solid electrode materials [7-10]. But the use of frequency dispersion measurements can also have a great potential for the study of complete electrochemical systems, such as batteries [11].

The advantage of measurements taken in the frequency domain over measurements in the time domain (i.e. pulse or step response measurements $[1,12]$ ) is that the frequency response can be described analytically, using an equivalent circuit as model. Time domain analysis often requires the approximation of complex functions, e.g. infinite summations of exponential functions. The circuit elements represent the various (macroscopic) processes involved in the transport of charge and mass. The dispersion relations for most equivalent circuit elements are very simple.

If the (complex) immittance diagrams show distinct features, which can easily be related to specific subcircuits of the equivalent circuit model, analysis become quite simple. Often this can be accomplished by graphical means, using a compass and a ruler. However, if the time constants of the respective subcircuits are close together, or if elements with a fractional (e.g. Warburg, or a CPE-type element [13-15], a more sophisticated analysis procedure is needed. As the variation of one circuit parameter can influence large parts of the frequency dispersion, all parameters must be adjusted simultaneously in order to obtain the optimum fit to the data.

Kleitz and Kennedy [6] have developed a special program for fitting two depressed and interfering semi-circles in the impedance plane, which are frequently encountered with solid electrolytes showing grain boundary dispersion. Tsai and Whitmore [16] have described a procedure for fitting a specific equivalent circuit to immittance data. Macdonald et al. $[17,18]$ have demonstrated the power of a more general NLLSF program, based on the Marquardt algorithm [19], in multi parameter fits of real and synthetic immittance data. 
The present paper describes a general NLLSF program for fitting an equivalent circuit response to frequency dispersion measurements (immittance data). The fit procedure incorporates the Marquardt algorithm as well. Many different equivalent circuits may be employed through the use of an unique Circuit Description Code (CDC). This code is also essential for the construction of a fast analytical derivatives routine. The NLLSF principle will be discussed briefly as it is readily available in the literature, see e.g. Bevington [20]. The CDC and the analytical derivatives routine are explained in separate sections. A short overview of possible circuit elements is also provided. The power of such a NLLSF procedure is demonstrated in multi parameter fits performed on synthetic and real data using the program "EQIVCT".

\section{Special notations}

In the following sections complex functions will be denoted by an asterisk, ${ }^{*}$. The real and imaginary part of a complex function will be marked with a single prime, ', and a double prime, ", respectively, e.g.:

$S^{*}(\omega)=S^{\prime}(\omega)+j S^{\prime \prime}(\omega)$,

where $j=\sqrt{-1}$. The frequency dispersion data can be given in the admittance representation, subscript $y$, or in the impedance representation, subscript $z$. As all equations in the description of the NLLSF procedure are valid for both the impedance and admittance representation, the use of the symbol $Y$ will stand for either representation (general: immittance). The measurement data set is represented by:

$Y_{i}^{*}=Y_{i}^{\prime}+j Y_{i}^{\prime \prime}, \quad i=1 \ldots N$.

The fitting function is represented by $Y^{*}\left(\omega, a_{1} \ldots a_{M}\right)$.In special cases this may be abbreviated to:

$Y^{*}\left(\omega, a_{m}\right)=Y^{\prime}(\omega)+j Y^{\prime \prime}(\omega), \quad m=1 \ldots M$

\section{Nonlinear least squares fit procedure}

In general a set of $N$ measurements, $\left(x_{i} y_{i}, i=1 \ldots . N\right)$ can be simulated with a function of the independent variable, $x$, and a set of adjustable parameters, $a_{1} \ldots a_{M}$.
The form of this function, $Y\left(x, a_{1} \ldots a_{M}\right)$ depends on the physical model used to explain the measurement set. As the data set will contain random and systematic errors, it can not be reproduced exactly by the simulation function. The best fit of this function is found through minimizing the sum $S$ of the (weighted) squares of the difference between the measured and the simulated data sets:

$S=\sum_{i=1}^{N} w_{i}\left[y_{i}-Y\left(x_{i}, a_{1} \ldots a_{M}\right)\right]^{2}$.

If the inverse variance, $1 / \sigma_{i}^{2}$, of the measurement is used as weight factor, $S$ becomes the goodness of fit parameter $\chi^{2}[20]$. When the function $Y\left(x, a_{1} \ldots a_{M}\right)$ is linear in the parameters $a_{m}$, this can be done by setting the partial derivatives, $\left(\partial S / \partial a_{m}\right)$, to zero. The resulting $M$ simultaneous equations can then be solved and the parameters evaluated.

If the function is not linear in $a_{m}$, as is often the case in frequency dispersion analysis, the problem is linearized by developing $Y\left(x, a_{1} \ldots a_{M}\right)$ in a Taylor series around a set of starting values $a_{m}^{0}$ :

$$
\begin{aligned}
& Y\left(x, a_{1} \ldots a_{M}\right)=Y\left(x, a_{1}^{0} \ldots a_{M}^{0}\right) \\
& \quad+\sum_{m=1}^{M} \frac{\partial Y\left(x, a_{1} \ldots a_{M}\right)}{\partial a_{m}} \delta a_{m}+\ldots
\end{aligned}
$$

The higher order terms in eq. (5) are ignored. Inserting eq. (5) in eq. (4) and setting the partial derivatives of $S$ with respect to the new set of variables, $\delta a_{m}$, to zero we obtain again a set of $\boldsymbol{M}$ simultaneous equations in $\delta a$. In matrix form:

$a \cdot \delta a=p$

with

$\alpha_{m k}=\sum_{i=1}^{N} w_{i} \frac{\partial Y\left(x_{i}, a_{1} \ldots a_{M}\right)}{\partial a_{m}} \frac{\partial Y\left(x_{i}, a_{1} \ldots a_{M}\right)}{\partial a_{k}}$

and

$\beta_{k}=\sum_{i=1}^{N} w_{i}\left[y_{i}-Y\left(x_{i}, a_{1} \ldots a_{M}\right)\right] \frac{\partial Y\left(x_{i}, a_{1} \ldots a_{M}\right)}{\partial a_{k}}$

with all derivatives taken in the point $a_{1}^{0} \ldots a_{M}^{0}$. The parameters, $\delta a_{m}$, can be found by multiplying both sides 
of eq. (6) with the inverse matrix of $\alpha, \epsilon=\alpha^{-1}$. The $\delta a_{m}$ are then found from

$\delta a_{m}=\sum_{k=1}^{M} \epsilon_{m k} \beta_{k}$.

As the higher order terms in the Taylor expansion have been ignored, this is only an approximate solution. The obtained values $\delta a_{m}$ can be used to construct a new (and better) set of starting values for $a_{m}^{0}$ :

$a_{m}^{\prime}=a_{m}^{0}+\delta a_{m}, \quad m=1 \ldots M$.

When the minimum in $S$ is approached upon successive iterations, the higher order terms in the expansion will vanish as the $\delta a_{m}$ will decrease rapidly. In practice the iteration procedure is stopped when the decrease in $S$, or the change in the parameters $a_{m}$, has become insignificant.

Close to the minimum in $S$ this non-linear least squares fit (NLLSF) method converges rapidly. The method is less suited, however, if the starting values of the parameters are far from their optimum values. In this region a gradient search, in which the path of the steepest descend is calculated, is more appropriate [20]. A combination of these two NLLSF methods has been described by Marquardt [19]. The diagonal terms of the curvature matrix, $\alpha_{m m}$, are multiplied by a factor $(1+\lambda)$. If $\lambda$ is large the gradient search prevails. When $\lambda$ is small a more analytical search is obtained. The fit procedure is started with a small value of $\lambda$, e.g., 0.01 . If on a successive iteration the value of $S$ decreases, $\lambda$ is also decreased. If $S$ would increase on an iteration, the value of $\lambda$ is increased and the iteration is redone with the same set of starting values of $a_{m}^{0}$ until $S$ decreases again.

The fitting of immittance data seems to require two simultaneous fitting functions as there are two sets of data, i.e. the real and the imaginary component of the dispersion. Both can be fitted at the same time by modifying eq. (4):

$$
\begin{aligned}
S & =\sum_{i=1}^{N} w_{i}\left\{\left[Y_{i}^{\prime}-Y^{\prime}\left(\omega_{i}, a_{1} \ldots a_{M}\right)\right]^{2}\right. \\
& \left.+\left[Y_{i}^{\prime \prime}-Y^{\prime \prime}\left(\omega_{i}, a_{1} \ldots a_{M}\right)\right]^{2}\right\},
\end{aligned}
$$

where $Y^{\prime}$ and $Y^{\prime \prime}$ represent the real and imaginary part of the immittance. The independent variable

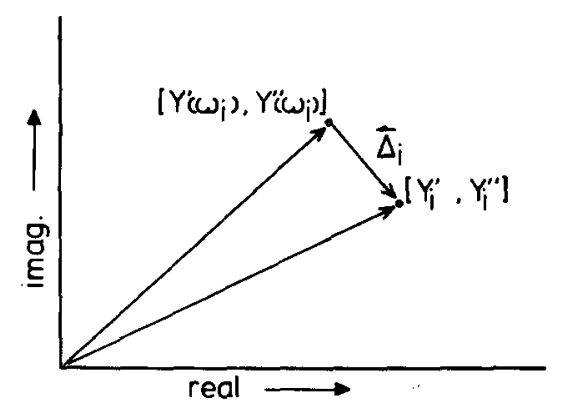

Fig. 1. Vector representation of the error function. In the fit procedure, the modules of the error vector $\Delta_{i}$ is minimized.

is the circular frequency $\omega$. Only one weight factor, $w_{i}$, is used as both data sets are not independent of each other. In fact, working out eq. (11) it can be seen that the sum of squares is merely the sum of the squares of the difference vectors, $\Delta_{i}$, between the measured and the calculated data sets (see fig. 1). In this respect there is only one fitting function.

An important choice in the fit procedure is the weight factor. Ideally the inverse of the variance of each measurement should be used. At low frequencies, however, it is very impractical to take several measurements at one frequency in order to establish the variance. Therefore the weight factors are replaced by a function which depends on the amplitude of the measurements. A reasonable choice is a function which lets each measurement contribute equally to the sum of squares. This is accomplished by taking the inverse of the square of the modulus of the immittance:

$w_{i}=1 /\left(Y_{i}^{\prime 2}+Y_{i}^{\prime \prime 2}\right)$.

This choice, which has also been proposed recently by Zoltowski [21], insures that the fit results are independent of the representation used (i.e. impedance or admittance representation).

An indication of the error in the resulting parameter values, $a_{m}$, can be obtained by assuming that the weight factors can be taken to be proportional to the inverse variances:

$w_{i}=g / \sigma_{i}^{2}$.

A further assumption is that the variances may be replaced by the parent distribution, in which case the reduced chi-squared, $\chi_{\nu}^{2}$, goes to unity: 


$$
\begin{aligned}
\chi_{\nu}^{2} & =\chi^{2} / \nu=\frac{1}{\nu} \sum_{i=1}^{N} \sigma_{i}^{-2}\left[y_{i}-Y\left(x_{i}, a_{1} \ldots a_{M}\right)\right]^{2} \\
& =S /(g \nu) \cong 1,
\end{aligned}
$$

where $\nu=N-M-1$ is the degrees of freedom of the system. With eq. (14) the value of $g$ can be obtained from the final value of $S$. The estimated uncertainties of the parameters, $\sigma_{a_{m}}^{2}$, follow then from the error matrix $\epsilon$, provided that the Marquardt parameter, $\lambda$, has been set to zero:

$\sigma_{a_{m}}^{2}=g \epsilon_{m m}$.

The covariance for the parameters can also be obtained from the error matrix [20].

\section{Calculation of the frequency response}

To make the NLLSF-program useful it must be able to accommodate different equivalent circuits. Of course, one can write the specific dispersion equations, describing the response of a particular equivalent circuit, in the program. However, if there is a need to amend the circuit, one has to rewrite these equations, with the risk of including programming errors. This is a rather inconvenient and time consuming route, as the program has then to be compiled and debugged again.

A more general approach would then be to devise a large array of elements and a corresponding set of dispersion relations, from which most equivalent circuits can be obtained by simply setting elements either to zero (short circuit) or infinity (open circuit). The disadvantage of this method is that many unproductive calculations have to be performed where elements have been removed from the array. This disadvantage is greater the lesser elements the equivalent circuit has.

The most general way would be to design a code, which uniquely represents a specific equivalent circuit, and which can be interpreted by the program in such a way that from a set of circuit parameters the proper response is calculated for each frequency. For a simple series or parallel circuit this could be done easily as the total circuit response is found by adding the individual responses of the elements in the proper representation. Thus for a series circuit of $Q$ elements: $\mathrm{E}_{1}, \mathrm{E}_{2}, \mathrm{E}_{Q}$ :
$S_{z}^{*}(\omega)=\sum_{k=1}^{Q} \mathrm{E}_{z k}^{*}(\omega)$,

where $S_{z}^{*}(\omega)$ is the overal circuit response in the impedance representation (denoted by $z$ ) at circle frequency $\omega . \mathrm{E}_{z k}^{*}(\omega)$ is the (complex) impedance of element $\mathrm{E}_{k}$ at frequency $\omega$. For a parallel circuit the circuit response is:

$S_{y}^{*}(\omega)=\sum_{k=1}^{Q} \mathrm{E}_{y k}^{*}(\omega)$,

where $y$ signifies the admittance representation, and

$\mathrm{E}_{y k}^{*}(\omega)=\left[\mathrm{E}_{z k}^{*}(\omega)\right]^{-1}$.

These additions can be performed by the computer program if the dispersion relations of the various types of elements (i.e. resistance, capacitance, Warburg etc.) are available in both representations in the program. Depending on the language used these could be written as functions (Pascal) or subroutines (Basic, Fortran). Calling these routines with the proper (adjustable) parameters, $a_{m}$, and frequency, $\omega_{i}$, then returns the required immittance value.

A circuit description code (CDC) can then be a string of symbols in which each symbol represents a specific type of element (e.g. $R$ for resistance, $W$ for Warburg). The code for a series (or parallel) circuit of elements $\mathrm{E}_{1}, \mathrm{E}_{2}, \mathrm{E}_{Q}$ would then become:

$\mathrm{E}^{1} \mathrm{E}^{2} \ldots \mathrm{E}^{Q}$,

where $E^{k}$ is the symbol representing the type of the element $\mathrm{E}_{k}$. In the program this symbol acts as a pointer to the specific routine which calculates the frequency dispersion of element $\mathrm{E}_{k}: \mathrm{E}_{x k}^{*}(\omega)$, where $x$ denotes impedance or admittance. The parameters of the elements must be in the same order as in which the $C D C$ is interpreted by the program. The overall response is then obtained for a series circuit in the impedance representation, following eq. (16), and for a parallel circuit in the admittance representation, according to eq. (17).

Most equivalent circuits, however, are more complex, containing several series and parallel subcircuits. In order to deal with this problem we define two sets of elements:

(1) A simple element is a two terminal element which represents a single (macroscopic) process, i.e. 
A

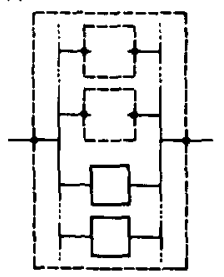

B

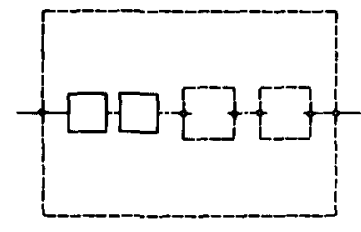

Fig. 2. Schematic representation of a composite element, (A): with a parallel internal subcircuit (odd level);(B): with a series subcircuit (even level). Solid lined boxes represent "simple" elements, broken lined boxes composite elements.

the corresponding transfer function cannot be reduced further in more fundamental transfer functions. The most common (simple) elements and their transfer functions are tabulated further in table 2 .

(2) A composite element is a two terminal element that contains either a series subcircuit or a parallel subcircuit, consisting of simple and/or composite elements (see fig. 2).

From this recursive definition it is obvious that the composite element, which is part of a series circuit of a next lower level composite element, must contain a parallel circuit, and vice versa. The nesting level of the subcircuit of a composite element is defined as the number of composite elements it is a part of. At the ground or zero level each complex circuit is a series circuit, thus it follows that composite elements of odd level always contain parallel subcircuits and those of even level always a series circuit.

With this set of definitions most equivalent circuits can be broken down into subcircuits of increasing nesting level. However, the two terminal requirement does impose a restriction on the circuits that can be represented by the CDC.

The occurrence of a composite element in the CDC is represented by a set of parenthesis. The left parenthesis, ( , signifies that the response of a complex element must be calculated. The current response summation, $S_{l}^{*}(\omega)$, at nesting level $l$ is suspended and a new response summation, $S_{l+1}^{*}(\omega)$, for the subcircuit of the composite element is started at level $l+1$. The end of the composite element calculation is signified by the right parenthesis, ). As the response sum, $S_{l+1}^{*}(\omega)$ is in the alternate representation of the lower level sum $S_{l}^{*}(\omega)$, it must be transformed before it can be added:

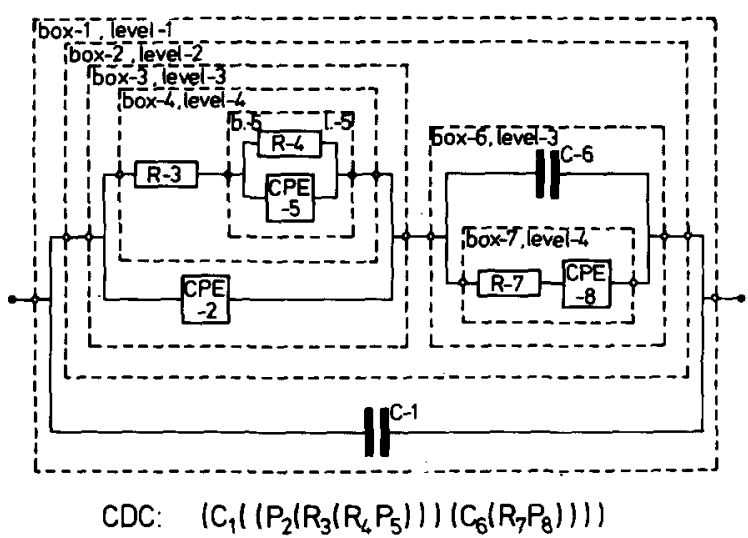

Fig. 3. Equivalent circuit used in ref. [22]. The broken lined boxes represent the composite elements in this equivalent circuit.

$S_{l}^{*}(\omega)_{\mathrm{new}}=S_{l}^{*}(\omega)+\left[S_{l+1}^{*}(\omega)\right]^{-1}$,

where

$\left[S_{l+1}^{*}(\omega)\right]^{-1}=\left\{S_{l+1}^{\prime}-j S_{l+1}^{\prime \prime}\right\} /\left\{S_{l+1}^{\prime 2}+S_{l+1}^{\prime \prime 2}\right\}$.

The subscript "new" in eq. (19) denotes the value of the response summation after adding the response of the composite element. This process of suspending calculations while an intermediate result has to be obtained first, is analogous to the algebraic notation used on many pocket calculators.

The development of the CDC for a complicated equivalent circuit is best done in consecutive steps. As an example the equivalent circuit of a previous publication [22] is used, see fig. 3. At the ground level there is only one composite element, outlined by box 1 . The parallel circuit in box-1 consists of a capacitance, $C$, and another composite element (box-2). This composite element contains a series circuit of two other composite elements (box-3 and box-6). Again these can be analysed, increasing the nesting level, but eventually only simple elements will remain. Then the circuit description code has been fully developed:

CDC: (C((P(R(RP)))(C(RP)))) .

The consecutive steps in this development are given in table 1. Fig. 4 shows schematically the actions taken upon interpretation of this $\mathrm{CDC}$ by the program. 
Table 1

Consecutive steps in the development of the circuit description code for the equivalent circuit of fig. 2 .

\begin{tabular}{|c|c|c|c|}
\hline Level & Circuit description & 2 code (CDC) & \\
\hline 0 & ( & box-1 & ) \\
\hline 1 & (c) & box-2 & ) \\
\hline 2 & icis & 3 )( box-6 & $5) 1$ \\
\hline 3 & (C) ( P ( box -4 & 4. )) (C (box-7) & 7)) ) \\
\hline 4 & ( C ( ( P (R (box -5$)$ & )$)(C(R P$ & ) \\
\hline 5 & $(C)(P(R(R))$ & ) )(C (RP & )1) \\
\hline
\end{tabular}

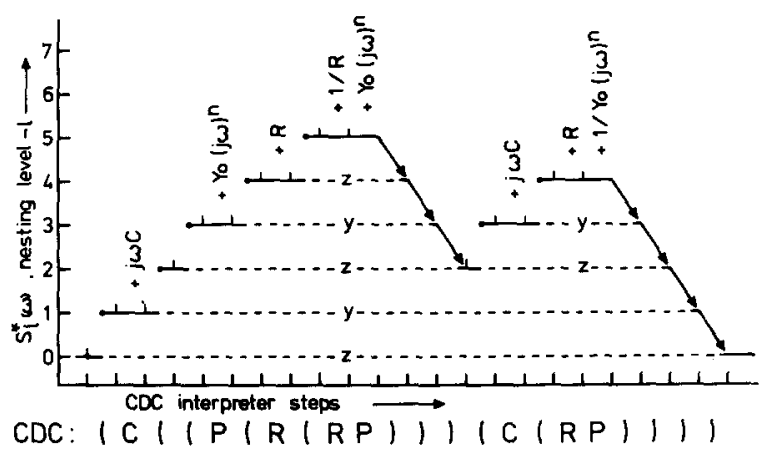

Fig. 4. Schematic representation of the steps taken in the interpretation of the CDC of fig. 3. The horizontal lines represent the subcircuit response summations, $S_{1}^{*}(\omega)$. A suspended summation is represented by a dashed line. The downward arrows symbolize the transformation and addition of the completed summation $S_{l}^{*}(\omega)$ to a lower level summation, $S_{l-1}^{*}(\omega)$ (eqs. $\left.(19,20)\right)$.

\section{Element representations and symbols}

The CDC symbols for the most common circuit elements, i.e. resistance: $R$, capacitance: $C$, and inductance: $L$ have been explained in the previous chapter. The corresponding dispersion relations are also well known. Four other, diffusion related, dispersion elements have currently been implemented in a NLLSfit program. The dispersion relations for three of these elements follow directly from the solution (in the frequency domain) of Fick's second law for one dimensional diffusion in mixed conducting materials. A schematic representation is given in fig. 5 . Here the admittance is defined as the current of mobile ions flowing through the mixed-conductor/electrolyte interface, divided by the voltage, measured at the inter-

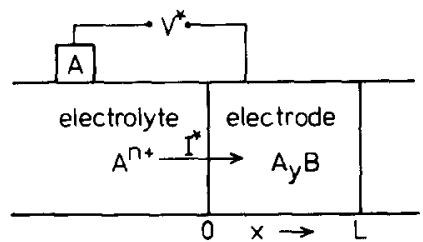

Fig. 5. Schematic diagram for one-dimensional diffusion in a mixed conducting electrode material, $A_{y} B$. The current, $I^{*}$, and the voltage, $V^{*}$, are complex, frequency dependent, variables.

face with respect to a separate (reference) electrode. The Nernst equation relates this voltage difference to the activity, and hence the concentration, of the mobile ion at the interface. For the three diffusional circuit elements different boundary conditions exist for the interface at $x=L$ (see fig. 5). Solutions in the frequency domain for these boundary value problems have been given recently by Honders and Broers [10] and by Raistrick and Huggins [23]. The most simple case is where the diffusion coefficient is small compared with the sample length $L$. For values of $\omega>$ $\widetilde{D} / L^{2}$ the semi-infinite Warburg diffusion is obtained:

$Y^{*}(\omega)=Y_{0} \sqrt{j \omega}=Y_{0} \sqrt{\omega / 2}+j Y_{0} \sqrt{\omega / 2}$.

In the case of a sample $A_{y} B$, with length $L$, interface area $s$, molar volume $V_{m}$, and chemical diffusion coefficient $\widetilde{D}$, see fig. $5, Y_{0}$ is given by [22]:

$Y_{0}=\frac{n F s \sqrt{\widetilde{D}}}{V_{m}}\left|\frac{\mathrm{d} E}{\mathrm{~d} y}\right|^{-1}$,

where $|\mathrm{d} E / \mathrm{d} y|$ is the slope of the electrochemical titration curve. If the boundary at $x=L$ is blocking for the mobile species a tangent hyperbolic function (CDC symbol "T") is obtained [10,23]:

$Y^{*}(\omega)=Y_{0} \sqrt{j \omega} \operatorname{Tanh}[B \sqrt{j \omega}]$

or separated in a real and imaginary component:

$$
\begin{aligned}
& Y^{*}(\omega)=Y_{0} \sqrt{\frac{\omega}{2}}\left[\frac{\sinh z-\sin z}{\cosh z+\cos z}\right. \\
& \left.+j \frac{\sinh z+\sin z}{\cosh z+\cos z}\right],
\end{aligned}
$$

where $z=B \sqrt{2 \omega} \cdot B$ contains the sample length and the diffusion coefficient:

$B=L / \sqrt{\widetilde{D}}$. 
For large values of $z(z \gg 1$, i.e. high frequencies) the Tanh-function becomes equal to 1 and eq. (24) can be replaced by the Warbug dispersion, eq. (22). For small values of $z(z \ll 1)$ the trigoniometric and hyperbolic functions must be replaced by their power series representations as their values become almost identical. Ignoring higher order terms, eq. (25) changes to:

$Y^{*}(\omega)=Y_{0} B^{3} \omega^{2} / 3+j Y_{0} B \omega$,

which is the admittance representation for a resistance $\left(R=B / 3 Y_{0}\right)$ in series with a capacitance $\left(C=Y_{0} B\right)$.

In the case that the interface at $x=L$ is non-blocking and imposes a fixed activity for the mobile species, as can be found for oxygen conducting electrodes [24] or in a symmetric arrangement of the cell of fig. 5 [10], a cotangent-hyperbolic function is obtained (CDC symbol 0):

$$
Y^{*}(\omega)=Y_{0} \sqrt{j \omega} \operatorname{coth}[B \sqrt{j \omega}] \text {. }
$$

Separated in a real and imaginary component:

$$
\begin{aligned}
& Y^{*}(\omega)=Y_{0} \sqrt{\frac{\omega}{2}}\left[\frac{\sinh z+\sin z}{\cosh z-\cos z}\right. \\
& \left.\quad+j \frac{\sinh z-\sin z}{\cosh z-\cos z}\right] .
\end{aligned}
$$

Again $z=B \sqrt{2 \omega}$, and $Y_{0}$ contains the same expression as in eq. (23). For large values of $z$ eq. (28) can be replaced by the Warburg representation, eq. (22). For small values of $z$ the same holds as for the Tanh function. Eq. (29) can then be approximated by

$Y^{*}(\omega)=Y_{0} / B+j Y_{0} B \omega / 3$,

which is the admittance representation of a resistance $\left(R=B / Y_{0}\right)$ parallel to a capacitance $\left(C=Y_{0} B / 3\right)$.

A generally observed dispersion relation is the Constant Phase Element or CPE [15], found in various electrochemical systems $[13,14]$. This element has a non-integer power dependence on the frequency:

$Y^{*}(\omega)=Y_{0}(j \omega)^{n}, \quad 0<n<1$.

Or separated in a real and imaginary part:

$Y^{\prime}=Y_{0} \omega^{n} \cos \frac{1}{2} n \pi, \quad Y^{\prime \prime}=Y_{0} \omega^{n} \sin \frac{1}{2} n \pi$.

Recently a theoretical derivation, based on the fractal geometry of electrode interfaces, has been given by Le Mehaute and Crepy [25]. Bates and Wang have shown that a non-integer power dependence can be obtained for a branched transmission line $[26,27]$. These cases deal with electrode/electrolyte interfaces only. However, the CPE dispersion has also been observed in the bulk response of ionic conductors. West et al. $[28,29]$ have investigated this type of bulk dispersion using the empirical "universal dielectric response" described by Jonscher [30]. In their curve fitting procedure they only regard the real component of the dispersion, without verifying whether the real and imagin. ary part still fulfill the Kramers-Kronig relation. This

Table 2

Dispersion equations and CDC-symbols for simple elements. For complete equations for the finite length diffusion element (Tanh and Coth), see text.

\begin{tabular}{llll}
\hline Element, symbol & \multicolumn{2}{l}{ Dispersion relation } \\
\cline { 2 - 3 } & admittance & impedance \\
\hline Resistance, $R:$ & $1 / R$ & $R$ \\
Capacitance, $C:$ & $j \omega C$ & $-j / \omega C$ \\
Inductance, $L:$ & $-j / \omega L$ & $j \omega L$ \\
Warburg, $\quad W:$ & $Y_{0} \sqrt{\omega / 2}(1+j)$ & $(1-j) / Y_{0} \sqrt{2 \omega}$ \\
CPE: $\quad P:$ & $Y_{0} \omega^{n}\left[\cos \frac{1}{2} n \pi+j \sin \frac{1}{2} \cdot n \pi\right]$ & $\omega^{n}\left[\cos \frac{1}{2} n \pi-j \sin \frac{1}{2} n \pi\right] / Y_{0}$ \\
Tanh. diff., $\quad T:$ & $Y_{0} \sqrt{j \omega} \operatorname{Tanh}[B \sqrt{j \omega}]$ & $\operatorname{Coth}[B \sqrt{j \omega}] / Y_{0} \sqrt{j \omega}$ \\
Coth. diff., $\quad 0:$ & $Y_{0} \sqrt{j \omega} \operatorname{Coth}[B \sqrt{j \omega}]$ & $\operatorname{Tanh}[B \sqrt{j \omega}] / Y_{0} \sqrt{j \omega}$ \\
\hline
\end{tabular}


method of analysis has recently been reviewed critically by Macdonald [31].

A summary of the CDC symbols and the dispersion relations is given in table 2. Other types of special elements can simply be added to the program by assigning an unique symbol to them and providing a subroutine in which the frequency response is calculated.

\section{Calculation of the derivatives}

Most NLLS-fit library programs need only an user written (sub-)routine for the calculation of the response of the fitting function $Y\left(x, a_{1} \ldots a_{M}\right)$. The derivatives with respect to the parameters $a_{m}$ of this function are obtained through a numerical evaluation. This is done by changing one parameter at a time by a small amount $\Delta a_{m}$, in both directions and calculating the corresponding change in the fitting function:

$\frac{\partial Y\left(x, a_{1} \ldots a_{M}\right)}{\partial a_{m}} \cong \frac{Y\left(x, a_{m}+\Delta a_{m}\right)-Y\left(x, a_{m}-\Delta a_{m}\right)}{2 \Delta a_{m}}$.

The disadvantage of this method is that the fitting function has to be evaluated $2 M$ times in order to obtain the set of $M$ partial derivatives. Besides, if the derivatives become very small, relative to $Y\left(x, a_{m}\right)$, the round-off errors in the computation become important.

Evaluating the derivatives analytically thus not only saves expensive computation time, but is also more precise. The derivatives of the frequency responses of the individual circuit elements, with respect to their adjustable parameters, can all be expressed analytically. These derivatives are tabulated in table 3 . How the partial derivatives of the total circuit response are obtained can be demonstrated by considering a simple element $\mathrm{E}_{k}$, which is part of the subcircuit of a composite element at level $l$. The partial derivative of the subcircuit response is then given by:

$\frac{\partial S_{l}^{*}(\omega)}{\partial a_{m}}=\frac{\partial \mathrm{E}_{k}^{*}(\omega)}{\partial a_{m}}$,

where $\mathrm{E}_{k}^{*}(\omega)$ represents the element dispersion function in the proper representation. $S_{l}^{*}(\omega)$ is the frequency response function of the composite element, which on its turn can be part of a subcircuit at level $l-1$. However, $S_{l}^{*}(\omega)$ is in the alternate representation of that of the lower subcircuit. The partial derivative of $S_{l-1}^{*}(\omega)$ with respect to $a_{m}$ becomes:

$$
\frac{\partial S_{l-1}^{*}(\omega)}{\partial a_{m}}=\frac{\partial\left[S_{l}^{*}(\omega)\right]^{-1}}{\partial a_{m}}=\frac{\partial}{\partial a_{m}} \frac{S_{l}^{\prime}-j S_{l}^{\prime \prime}}{S_{l}^{\prime 2}+S_{l}^{\prime \prime 2}},
$$

which can be separated into a real and imaginary component :

$$
\frac{\partial S_{l-1}^{\prime}}{\partial a_{m}}=\frac{S_{l}^{\prime 2}-S_{l}^{\prime 2}}{\left[S_{l}^{\prime 2}+S_{l}^{\prime 2}\right]^{2}}\left[\frac{\partial S_{l}^{\prime}}{\partial a_{m}}\right]-\frac{2 S_{l}^{\prime} S_{l}^{\prime \prime}}{\left[S_{l}^{\prime 2}+S_{l}^{\prime 2}\right]^{2}}\left[\frac{\partial S_{l}^{\prime \prime}}{\partial a_{m}}\right]
$$

and

$$
\frac{\partial S_{l-1}^{\prime \prime}}{\partial a_{m}}=\frac{S_{l}^{\prime \prime 2}-S_{l}^{\prime 2}}{\left[S_{l}^{\prime 2}+S_{l}^{\prime 2}\right]^{2}}\left[\frac{\partial S_{l}^{\prime \prime}}{\partial a_{m}}\right]+\frac{2 S_{l}^{\prime} S_{l}^{\prime \prime}}{\left[S_{l}^{\prime 2}+S_{l}^{\prime \prime 2}\right]^{2}}\left[\frac{\partial S_{l}^{\prime}}{\partial a_{m}}\right] \text {. }
$$

Eqs. (34)-(36) describe how the response derivatives of an element are transformed while stepping down through the corresponding nesting levels of that element. The partial derivatives of the total circuit response, $Y^{*}\left(\omega, a_{1} \ldots a_{M}\right)$, (in the impedance representation) is obtained when the derivative $\left[\partial S_{0}^{*}(\omega) / \partial a_{m}\right]$ has been evaluated. One more transformation gives the derivatives in the admittance representation.

The calculation of the derivatives involves the subcircuit response summations, $S_{l}^{*}(\omega)$, which have already been calculated in the circuit response evaluation. Storing these values in a set of arrays during the response calculation saves computing time. This storage requires, however, a large amount of memory. The derivatives of the various elements (see table 3 ) can be introduced in the program as subroutines or functions. A set of pointers to those routines can be derived from the CDC. From the CDC also a sequence of transformations (eqs. (35-37)) is derived for each element. These sequences contain pointers to the proper subcircuit response summations, $S_{l}^{*}(\omega)$, which are needed for the calculation of the partial derivatives of the circuit response. It is also possible to calculate the derivatives together with the calculation of the equivalent circuit response. In that case the derivative equations can be enclosed in the respective element response routines, see section 4 . 


\section{Table 3}

Derivatives of the "simple" elements. If a parameter would appear in the denominator of a dispersion equation, it is replaced by its inverse, thus simplifying the equation of the derivative. $Y^{*}(\omega)$ and $Z^{*}(\omega)$ represent the dispersion formulae of the elements in the admittance and impedance representation respectively, see table 2.

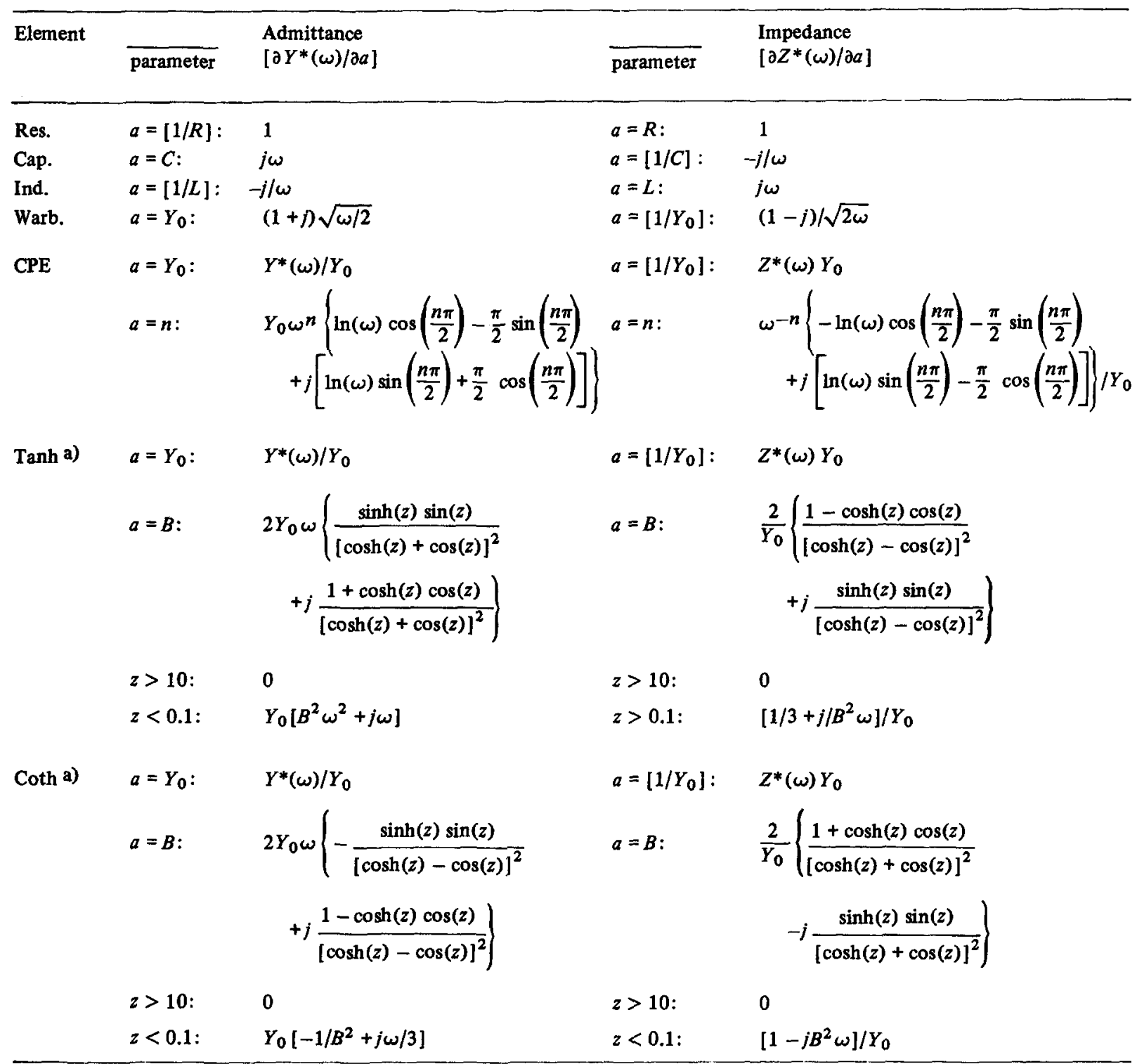

a) $z=B \sqrt{2 \omega}$.

\section{Simulated data test}

The power of the NLLSF-program has previously been shown [22] with an eleven parameter fit based on the equivalent circuit of fig. 3 . The actual param- eter values for real data cannot be known, which makes it impossible to check whether the fit parameters are appropriate. Therefore synthetic data has been constructed using the parameter values as obtained in the NLLS-fit of a frequency dispersion mea- 
Table 4

Result of a fit performed on synthetic data with the NLLSF program "EQIVCT". The data was constructed with the parameter set of the first column. The second column presents the starting values obtained in a preliminary analysis after noise has been added to the data. The NLLS-fit results, together with the estimated uncertainties, are presented in the last column.

\begin{tabular}{|c|c|c|c|c|c|c|c|}
\hline \multirow{2}{*}{\multicolumn{2}{|c|}{ Element }} & \multicolumn{2}{|c|}{ Actual value } & \multicolumn{2}{|c|}{ Start value } & Fit result & Rel. err. \\
\hline & & 2.8 & $\times 10^{-12}$ & 3.2 & $\times 10^{-12}$ & $2.78 \times 10^{-12}$ & $5 \%$ farad \\
\hline \multirow{2}{*}{${ }^{C P E}-2\left\{\begin{array}{l}Y_{0} \\
n\end{array}\right.$} & : & 7.2 & $\times 10^{-10}$ & 9.9 & $\times 10^{-10}$ & $6.98 \times 10^{-10}$ & $10 \%$ mho \\
\hline & : & \multicolumn{2}{|c|}{0.62} & \multicolumn{2}{|c|}{0.59} & 0.623 & $1.5 \%$ \\
\hline resistance-3 & : & \multicolumn{2}{|c|}{$7.82 \times 10^{5}$} & \multicolumn{2}{|c|}{7.8} & $7.85 \times 10^{5}$ & $0.5 \% \mathrm{ohm}$ \\
\hline resistance 4 & : & 1.61 & $\times 10^{?}$ & 7.6 & $\times 10^{5}$ & $1.62 \times 10^{7}$ & $1.2 \% \mathrm{ohm}$ \\
\hline \multirow{2}{*}{ CPE-5 $\left\{\begin{array}{l}Y_{0} \\
n\end{array}\right.$} & : & 3.35 & $\times 10^{-8}$ & 3.6 & $\times 10^{-8}$ & $3.37 \times 10^{-8}$ & $0.7 \% \mathrm{mho}$ \\
\hline & : & \multicolumn{2}{|c|}{0.705} & \multicolumn{2}{|c|}{0.69} & 0.704 & $0.3 \%$ \\
\hline capacitance-6 & $\because$ & 2.5 & $\times 10^{-7}$ & 3.0 & $\times 10^{-8}$ & $2.56 \times 10^{-7}$ & $2 \%$ farad \\
\hline resistance-7 & $:$ & 2.2 & $\times 10^{7}$ & 1.6 & $\times 10^{7}$ & $2.29 \times 10^{7}$ & $3 \%$ ohm \\
\hline \multirow[t]{2}{*}{$\mathrm{CPE}-8, Y_{0}$} & $:$ & 2.1 & $\times 10^{-7}$ & 2.4 & $\times 10^{-7}$ & $2.12 \times 10^{-7}$ & $1.6 \%$ mho \\
\hline & $:$ & \multicolumn{2}{|c|}{0.70} & \multicolumn{2}{|c|}{0.70} & 0.703 & $0.5 \%$ \\
\hline
\end{tabular}

surement on the ionic conductor $\mathrm{Na}_{2} \mathrm{SnS}_{3}$ [22]. The program used for generating the data set was based on the structure as described in section 4. It was written in Basic and runs on a personal computer (48k Apple II) [32]. Subsequently the data was polluted with random noise with a Gaussian distribution between $-3 \sigma$ and $+3 \sigma$, where $\sigma^{2}$ was taken as $0.3 \%$ of the modulus $\sigma_{i}^{2}=0.003 \times\left[\left(Y_{i}^{\prime}\right)^{2}+\left(Y_{i}^{\prime \prime}\right)^{2}\right]^{1 / 2}$.

With this data set a preliminary analysis was performed in order to find a set of starting values for the circuit parameters. This was achieved with a very simple Basic program in which the individual responses of the circuit elements can be subtracted from the circuit response. The simulated data set was transferred to the main frame computer (CDC Cyber 170-760) and analyzed with the NLLSF-program "EQIVCT". Weight factors according to eq.(11) were used. The results of this fit procedure, which took 15 iterations $(1.3 \mathrm{~s}$ in machine time) are shown in table 4 , together with the set of starting values and the actual circuit parameters. It can be seen that despite the complexity of the circuit and the number of parameters involved, a very good fit is obtained. The quality of the parameter fit of an equivalent circuit to a set of immittance data can best be seen in a Fit Quality Plot (FQ-plot) [32] in which the relative deviations of the real, $\Delta_{\mathrm{Re}}$, and imaginary part, $\Delta_{\mathrm{Im}}$, of the immittance are plotted against $\log (\omega)$ :

$$
\Delta_{\mathrm{Re}_{i}}=\frac{Y_{i}^{\prime}-Y^{\prime}\left(\omega_{i}\right)}{\left|Y_{i}^{*}\right|} \text { and } \Delta_{\mathrm{Im}_{i}}=\frac{Y_{i}^{\prime \prime}-Y^{\prime \prime}\left(\omega_{i}\right)}{\left|Y_{i}^{*}\right|}
$$

For a good fit these relative deviations should be randomly distributed around the horizontal $\log (\omega)$ axis, as is demonstrated in fig. 6 .

The correlation between the 11 parameters was found to be very large. This is because the CPE elements influence the frequency dispersion over a large frequency range, and the respective time constants are rather close together.

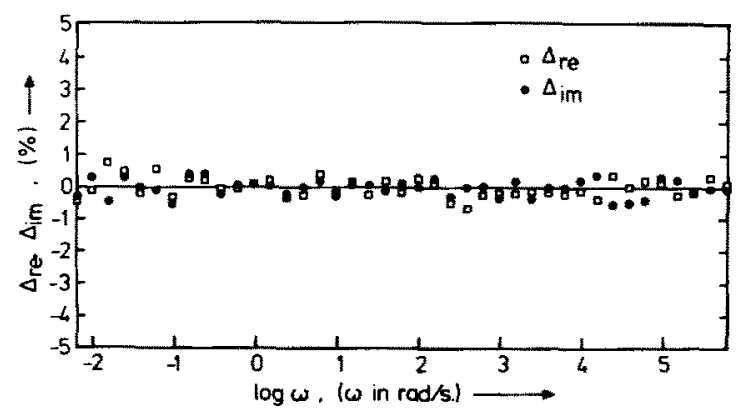

Fig. 6. FQ-plot for the NLLS-fit of the equivalent circuit of fig. 3 to a set of synthetic data. 


\section{NLLS-fit of ac-diffusion data}

The insertion of mobile inns in mixed conducting materials can be studied quite efficiently with the combination of electrochemical titration and frequency dispersion measurements, using a three electrode cell $[7,10,33]$. An example of the NLLSF analysis of an ac-diffusion measurement on the mixed conducting layer compound $\mathrm{Ag}_{x} \mathrm{NbS}_{2}$ is presented here. The frequency response measurements were performed using a micro computer for signal analysis and data acquisition. The experimental set up and electrochemical cell arrangement have been described elsewhere $[8,22]$.

The measured frequency dispersion shown in fig. 7 (open circles), is a typical example for these types of measurements. From first observation, the dispersion could be represented by an electrolyte resistance in series with the finite length diffusion element (Tanhfunction, eqs. (24-27)). A randless type circuit is generally not observed in these all-solid electrochemical cells $[8,33]$. From previous experiments, however, it was noted that the measuring system contributes an inductive dispersion to the measured frequency disper-

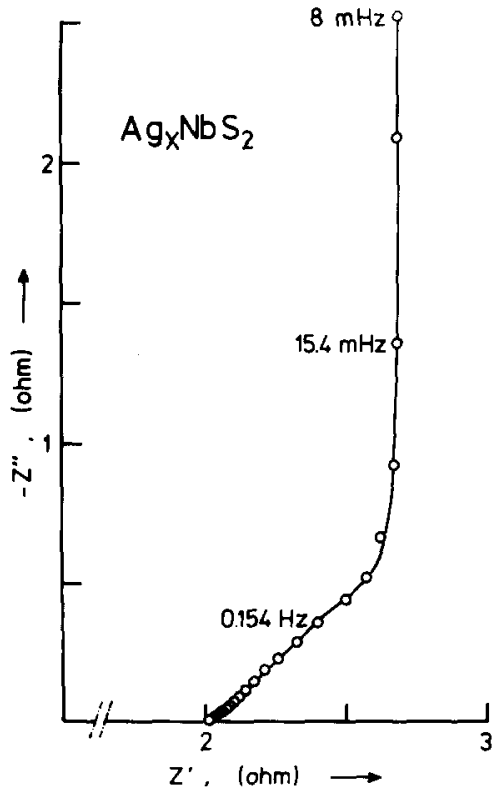

Fig. 7. Typical example of the frequency dispersion of the impedance of a mixed conducting electrode material. Open circles represent data points obtained from an $\mathrm{Ag}_{x} \mathrm{NbS}_{2}$ sample [9]. The solid line represents the fit result.
Table 5

Parametex fit of ac-diffusion measurement. Two different equivalent circuits were used. The relative uncertainties are given in \%. An asterisk indicates that the uncertainty was very large, due to a strong correlation between parameters.

\begin{tabular}{|c|c|c|c|c|c|c|}
\hline \multicolumn{4}{|c|}{ Circuit code: $L R(R P) T$} & \multicolumn{3}{|l|}{$R T$} \\
\hline \multicolumn{7}{|l|}{ Element: } \\
\hline & $L:$ & $4 \times 10^{-5}$ & $*$ & & & $\mathbf{H}$ \\
\hline & $R:$ & 1.26 & $*$ & 2.03 & $0.5 \%$ & ohm \\
\hline & $R:$ & 0.78 & $*$ & & & \\
\hline \multirow[t]{2}{*}{ CPE } & $Y_{0}:$ & $2.1 \times 10^{-3}$ & $*$ & & & mho \\
\hline & $I_{n:}$ & 0.53 & $*$ & & & \\
\hline \multirow[t]{2}{*}{ Tanh } & $Y_{0}:$ & 2.02 & $0.5 \%$ & 1.99 & $0.5 \%$ & mho \\
\hline & $\hat{l}_{B:}$ & 3.97 & $0.5 \%$ & 4.01 & $0.6 \%$ & \\
\hline
\end{tabular}

sion. The corresponding inductance was estimated at $30 \mu$ Henri. Also sometimes a diffusional reaction layer developed between the sample and the electrolyte. This can be represented in the equivalent circuit by a CPE in parallel with a resistance. Therefore several NLLSF-fits were performed with various equivalent circuits. Only two circuits showed a good fit to the data. The resulting parameter values are presented in table 5. The two element circuit, CDC: "RT", showed a small trace in the FQ-plot at the high frequency end, while the circuit with CDC: "LR(RP)T" showed an excellent fit in the FQ-plot, see fig. 8. The estimated

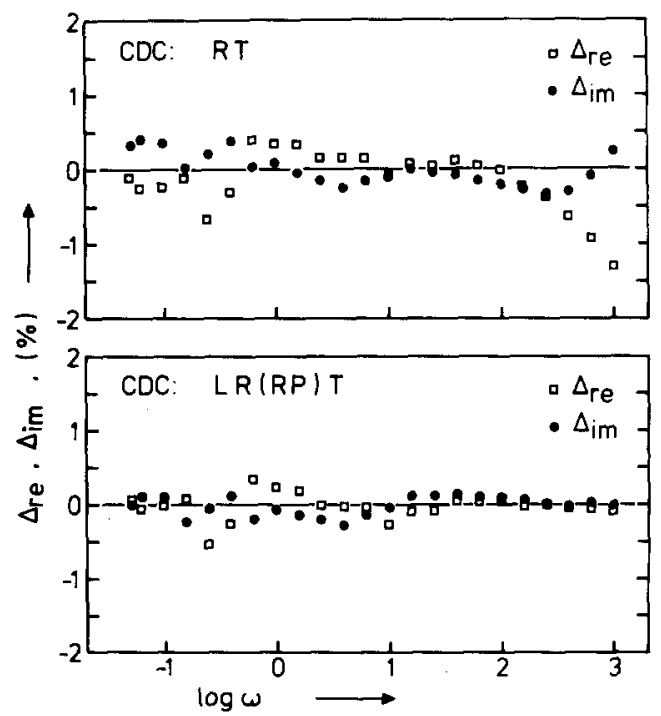

Fig. 8. FQ-plots for the NLLS-fit results for the ac-diffusion data of fig. 7 , using two different equivalent circuits. 
uncertainties in the latter fit are rather high, which is due to a very strong correlation between some of the circuit parameters. The observed inductance value is quite close to the previously estimated value. From this analysis it may be concluded that the reaction layer is present, but no reliable parameter values can be obtained. The parameters of the Tanh-function are not affected by the different analysis, as might be expected from the shape of the frequency dispersion.

\section{Discussion}

The nonlinear least squares fit technique is a very valuable tool for analyzing complex frequency dispersion data. Although these fitting procedures are readily available from computer libraries (e.g. the NAG or IMSL libraries) it is worthwhile to write ones own fit program following the route described in this paper. The great advantage of the response calculation based on a circuit description code (section 4) is that once the separate dispersion formulae of the various types of circuit elements have been written correctly in the program, a change in the equivalent circuit is implemented without rewriting parts of the program.

The use of an analytical derivatives routine (section 6) instead of the numerical evaluation method employed by the library fit programs, not only enhances the precision of the fit but also dramatically increases the speed of execution of the program. This is a significant advantage as computing time on main frame computers is quite costly.

In this respect it is useful to consider the optimum number of immittance data per dispersion measurement. Although a large number of measurements per (frequency) decade seems a good choice for obtaining the best fit, one should realize that computing time increases linearly with the number of data sets. Thus it is more appropriate to invest measurement time in obtaining a minimal number of data points but with a maximum reliability. In practice it appears that 5 to 7 steps per decade are sufficient for an optimal fit result. Using white noise and a FFT spectrum analyser for frequency dispersion measurements [34] is less suited for a subsequent NLLSF analysis, as this method yields a great number of data sets with rather low precision.

To ensure a good fit result the frequency disper- sion data must be free of systematic errors. One source of distortion of the dispersion data is the input impedance of the measuring instrument together with possible internal frequency dependent signal gains and phase shifts. With the use of small computers in the data acquisition system, the measurements can easily be corrected for these type of errors [22]. Also the temperature of the measurement cell should be very constant. Assuming an Arrhenius type behaviour for the parameter $a_{m}$ of an element, the relative change with a change $\Delta T$ in temperature can be expressed as:

$$
\left[\Delta a_{m} / a_{m}\right]=\Delta T \cdot H / k \cdot T^{2}
$$

which at $300 \mathrm{~K}$ and with an activation enthalpy of $100 \mathrm{~kJ} / \mathrm{mole}(1.04 \mathrm{eV})$ gives $\left[\Delta a_{m} / a_{m}\right]=0.13 \times \Delta T$, or a $13 \%$ change for 1 degree increase in temperature.

If the system parameters change continuously with time, a NLLS-fit may still be used after the data has been transformed to a "constant time" data set. Such a scheme has recently been presented by Stoynov and Savova-Stoynov $[35,36]$. In this interesting procedure the time is an extra dimension in the frequency dispersion measurement. In the time-immittance frame, constant frequency projections are created through fitting of cubic spline functions to the immittance-time data sets. From these functions a set of frequency-immittance projections at constant time can be obtained, which subsequently can be analyzed with a NLLSF procedure.

The choice for the set of starting parameters, eq. (5), is very important, as the number of iterations in the fit procedure will depend sharply on the "quality" of this set. Using a personal computer the data set can be analyzed in a crude manner by subtracting the dispersions of elements or combinations of elements from the total frequency distribution [32], or by simple graphical means. The choice of the proper equivalent circuit is crucial to a good fit result. Over parameterisation is not very likely here, as only a limited number of very distinct dispersion relations are allowed. The parameters of superfluous circuit elements will be pushed either to zero or infinity by the fit procedure, whereas insufficient elements will result in an obviously bad fit, which will show a distinct trace in ti.e FQ-plot.

Of course one should be aware of the equivalence of two special circuits [37], see fig. 9. If circuit (a) 


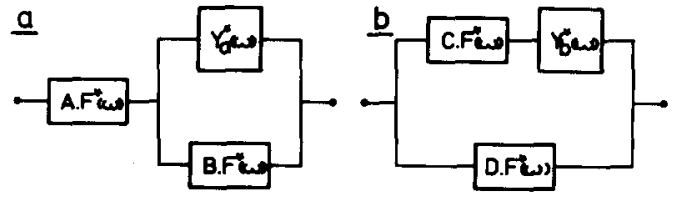

Fig. 9. Two equivalent circuits which can have exactly identical frequency dispersions.

contains two elements with the same frequency dispersion, $A . F^{*}(\omega)$ and $B . F^{*}(\omega)$, an alternate circuit, fig. $9(\mathrm{~b})$, with similar elements C.F* $(\omega)$ and D.F $(\omega)$, can be constructed to give exactly the same frequency response. The dispersion functions here are in the admittance representation. The relation between the parameters of the circuit elements is then given by:

$\mathrm{C}=\mathrm{A} /[\mathrm{A}+\mathrm{B}]$ and $\mathrm{D}=\mathrm{A} \cdot \mathrm{B} /[\mathrm{A}+\mathrm{B}]$

and

$Y_{\mathrm{b}}^{*}(\omega)=Y_{\mathrm{a}}^{*}(\omega) /[1+\mathrm{B} / \mathrm{A}]^{2}$,

where $Y^{*}(\omega)$ can be any type of circuit. Which of the two circuits correctly represents the electrochemical processes can only be elucidated through variation of the circuit parameters, e.g. measurements at different temperatures.

With the information provided in this paper, an experienced programmer should be able to construct a similar NLLSF program for immittance data analysis. The source code (FORTRAN 77) of the NLLSF program "EQIVCT" will be made available upon request.

\section{Acknowledgement}

The major part of this study was performed at the State University of Groningen, Department of Inorganic Chemistry. The author wishes to thank Dr.

Wiegers for his stimulating advise and attention during the development of the NLLSF program and for carefully reading the manuscript. Dr. Bouwmeester is thanked for providing the set of ac-diffusion measurements.

\section{References}

[1] P.H. Bottelberghs, in: Solid electrolytes, eds. P. Hagenmuller and W. van Gool (Academic Press, New York, 1978) p. 145.

[2] J.R. Macdonald and J.A. Garber, J. Electrochem. Soc. 124 (1977) 1022.

[3] I.D. Raistrick and R.A. Huggins, in: Proc. Symposium and Workshop on Advanced Battery Research and Design, Argonne National Laboratory 76-8 (1976) B277.

[4] T.M. Gür, I.D. Raistrick and R.A. Huggins, in: Fast ion transport in solids, eds. P. Vashishta, J.N. Mundy and G.K. Shenoy (North-Holland, Amsterdam, 1979) p. 113.

[5] D.R. Franceschetti, J. Schoonman and J.R. Macdonald, Solid State Ionics 5 (1981) 617.

[6] M. Kleitz and J.H. Kennedy, in: Fast ion transport in solids, eds. P. Vashishta, J.N. Mundy and G.K. Shenoy (North-Holland, Amsterdam, 1979) p. 185.

[7] C. Ho, I.D. Raistrick and R.A. Huggins, J. Electrochem. Soc. 127 (1980) 343.

[8] B.A. Boukamp and G.A. Wiegers, Solid State Ionics 9/10 (1983) 1193.

[9] H.J.M. Bouwmeester, G.A. Wiegers and B.A. Boukamp, in: Proc. Int. Conf. Solid State Batteries, NASI 1984 (Alcabedeche, Portugal), to be published.

[10] A. Honders and G.H.J. Broers, Solid State Ionics 15 (1985) 173.

[11] R. Mauger, M. Elkordi, J.C. Pariaud, F. Dalard and D. Deroo, J. Appl. Electrochem. 14 (1984) 293.

[12] W. Weppner and R.A. Huggins, Ann. Rev. Mater. Sci. 8 (1978).

[13] P.H. Bottelberghs and G.H.J. Broers, J. Electroanal. Chem. 67 (1976) 155.

[14] I.D. Raistrick, C. Ho and R.A. Huggins, J. Electrochem. Soc. 123 (1976) 1469.

[15] J.R. Macdonald, Solid State Ionics 13 (1984) 147.

[16] Y.T. Tsai and D.H. Whitmore, Solid State Ionics 7 (1982) 129.

[17] J.R. Macdonald, A. Hooper and A.P. Lehnen, Solid State Ionics 6 (1982) 65 .

[18] I.R. Macdonald, J. Schoonman and A.P. Lehnen, J. Electroanal. Chem. 131 (1982) 77.

[19] D.W. Marquardt, J. Soc. Ind. Appl. Math. 11 (1963) 431.

[20] P.R. Bevington, Data reduction and error analysis for the physical sciences (McGraw-Hill, New York, 1969).

[21] P. Zoltowski, J. Electrochem. Chem. 178 (1984) 11.

[22] B.A. Boukamp, Solid State Ionics 11 (1984) 339.

[23] I.D. Raistrick and R.A. Huggins, Solid State Ionics 7 (1982) 213.

[24] G.H.J. Broers and M. Schenke, in: Proc. Int. Conf. on Fuel Cells (Akademie Verlag, Dresden, 1967) p. 229.

[25] A. Le Mehaute and G. Crepy, Solid State Ionics 9/10 (1983) 17 
[26] J.B. Bates, J.C. Wang and R.L. Anderson, ECS Fall Meeting (New Orleans, 1984). Extended Abstracts, Vol. 84-2, p. 233.

[27] J.C. Wang and J.B. Bates, ECS Fall Meeting (New Orleans, 1984) Extended Abstracts Vol. 84-2, p. 234

[28] P.G. Bruce, A.R. West and D.P. Almond, Solid State Ionics 7 (1982) 57.

[29] D.P. Almond and A.R. West, Solid State Ionics 9/10 (1983) 277.

[30] A.K. Jonscher, Phys. Thin Films 11 (1980) 202.

[31] J.R. Macdonald, Solid State Ionics 15 (1985) 159.
[32] B.A. Boukamp, Solid State Ionics 18/19 (1986) 136.

[33] A.G. Gerards, H. Roede, R.J. Haange, B.A. Boukamp and G.A. Wiegers, Synth. Metals $10(1984 / 85) 51$.

[34] A. Chabli, J.P. Diard, P. Landaud and B. le Gorrec, Electrochim. Acta 29 (1984) 509.

[35] B.S. Savova-Stoynov and Z. Stoynov, Electrochem. Soc. $85-3$ (1985) 152 .

[36] Z.B. Stoynov and B.S. Savova-Stoynov, J. Electroanal. Chem. 183 (1985) 133.

[37] J.R. Macdonald, in: Electrode processes in solid state ionics, eds. M. Kleitz and J. Dupuy (Reidel, Dordrecht, 1976) p. 149. 